\title{
Field Sampling from a Segmented Image *
}

\author{
Pravesh Debba ${ }^{12 \star \star}$, Alfred Stein ${ }^{3}$, Freek D. van der Meer ${ }^{3}$, Emmanuel John M. \\ Carranza $^{3}$, and Arko Lucieer ${ }^{4}$ \\ 1 University of South Africa, College of Science, Engineering and Technology, \\ Department of Statistics, P. O. Box 392, Pretoria, 0003, South Africa, \\ debbap@unisa.ac.za \\ 2 Council for Scientific and Industrial Research (CSIR), Logistics and Quantitative \\ Methods, CSIR Built Environment, P. O. Box 395, 0001, South Africa. \\ pdebba@csir.co.za \\ 3 International Institute for Geo-Information Science and Earth Observation (ITC), \\ Hengelosestraat 99, PO Box 6, 7500AA Enschede, The Netherlands \\ 4 University of Tasmania, School of Geography \& Environmental Studies, Center for \\ Spatial Information Science (CenSIS), Private Bag 76, Hobart, Tasmania, 7001, \\ Australia
}

\begin{abstract}
This paper presents a statistical method for deriving the optimal prospective field sampling scheme on a remote sensing image to represent different categories in the field. The iterated conditional modes algorithm (ICM) is used for segmentation followed by simulated annealing within each category. Derived field sampling points are more intense in heterogenous segments. This method is applied to airborne hyperspectral data from an agricultural field. The optimized sampling scheme shows superiority to simple random sampling and rectangular grid sampling in estimating common vegetation indices and is thus more representative of the whole study area.
\end{abstract}

\section{Introduction}

Sampling entails the selection of a part of a population to draw inference about the whole population. Random sampling is attractive as it implies unbiasedness, resulting in independent observations, which are a basic requirement for statistical inference. In geological and vegetational studies, though, it may conflict with the desire for representativeness. For example, points could be confined to only one part of the area of interest and specific local features may be missed. Geostatistical data often show spatial autocorrelation, hence random sampling may no longer be optimal and for some purposes equally spaced samples or clustered samples are more useful. In addition, estimation of the population mean may benefit from partitioning into homogeneous strata [33, 21,31].

\footnotetext{
* This work was sponsored by ITC International Institute for Geo-Information Science and Earth Observation, project number 3083022 and NRF National Research Foundation, project number 10317, gun 2053944.

** Corresponding author: Tel: +27-12-4296531 Fax: +27-12-4296298
} 
Segmented images have various applications. In this study the design of the optimal prospective sampling scheme is considered. Design of prospective sampling schemes for classification using ground truth samples has been problematic in geological and vegetational studies. In the past samples have been randomly selected aided with some form of disciplinary judgement [20,5]. High spatial and spectral resolution hyperspectral imagery now makes it possible to select sampling locations in advance of fieldwork. Such images provide a synoptic overview of a large area and often provide topographic information that are more accurate and detailed than ground truth maps [9].

In context of vegetational studies, biophysical parameters, such as leaf area index (LAI), biomass, chlorophyll concentration, and photosynthetic activity, are important for estimating foliage cover and forecasting vegetation growth and yield (e.g., [16]). By selecting appropriate bands, a segmented image can reflect spatial information of variability of certain biophysical parameters, and one could potentially optimize field visits to better estimate these parameters of interest.

This paper considers the design of the optimal prospective sampling scheme for field visits in an agricultural study, using a segmented hyperspectral image. The optimal prospective sampling scheme will be representative of the whole study area for various parameters embedded by the segmentation and bands selected for the segmentation. The paper is illustrated with airborne hyperspectral data, DAIS-7915 acquired over the Tedej area in Hungary.

\section{Study Site}

\subsection{Study Area}

Tedej, Hajdu-Bihar area, Hungary (see Figure 1), approximately 1500 ha, is an intensively cultivated agricultural land neighboring a natural protection park area [17]. Soil categories characteristic to this area are Chernozems, Phaeozems, Solonchaks and Solonetz [17] and major crops are barley, maize, sugar beet, sunflower and alfalfa.

This study area also includes non-vegetation areas (cultivated areas and pathways between fields), and as such was ideal for constraining the sampling. An appropriate sampling scheme, representative of the different crop categories, consist of samples distributed evenly over the respective categories of interest, at the same time avoiding the boundaries of crop categories because of the higher levels of uncertainty at the boundaries.

\subsection{Remote Sensing}

In this study, a subset of the Digital Imaging Spectrometer (DAIS-7915), is used. The resulting data is a 79 channel hyperspectral image that was acquired over the Tedej area (see Figure 2). DAIS-7915 is a whisk broom sensor, covering a spectral range from visible $(0.4 \mu \mathrm{m})$ to thermal infrared $(12.3 \mu \mathrm{m})$ at variable 


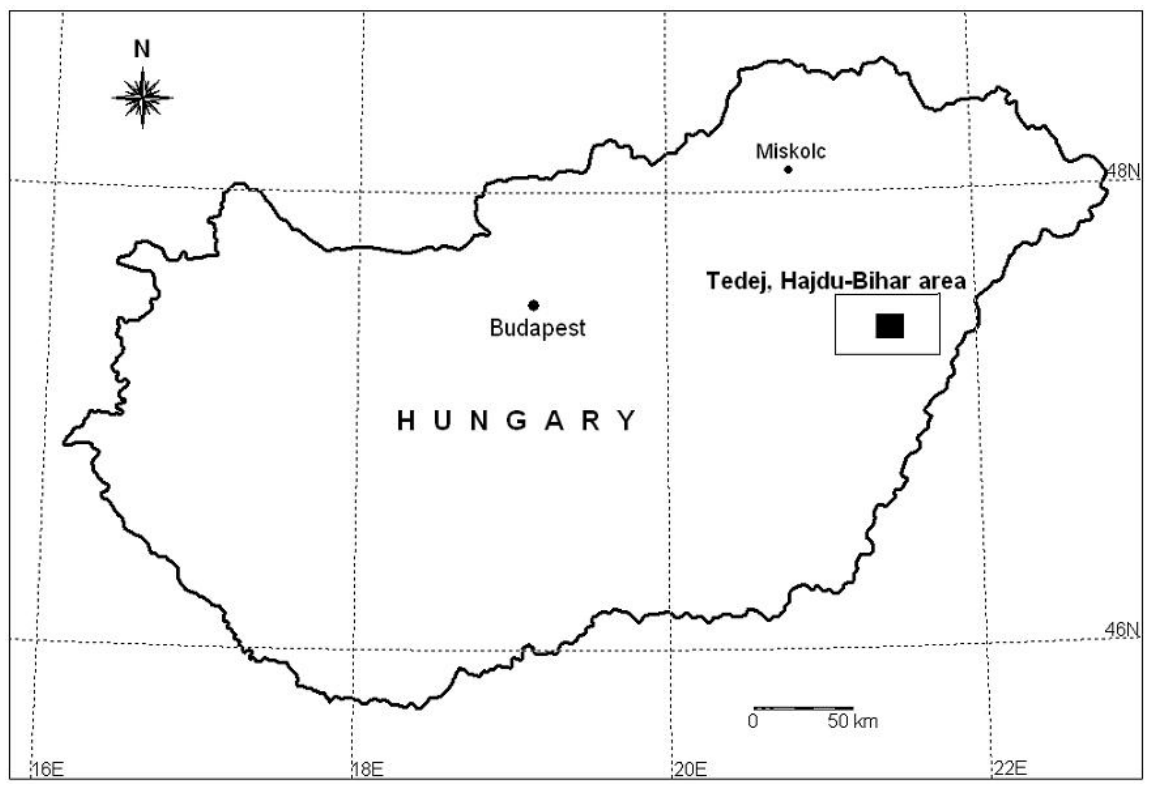

Fig. 1. Study area in Tedej, Hajdu-Bihar area, Hungary.

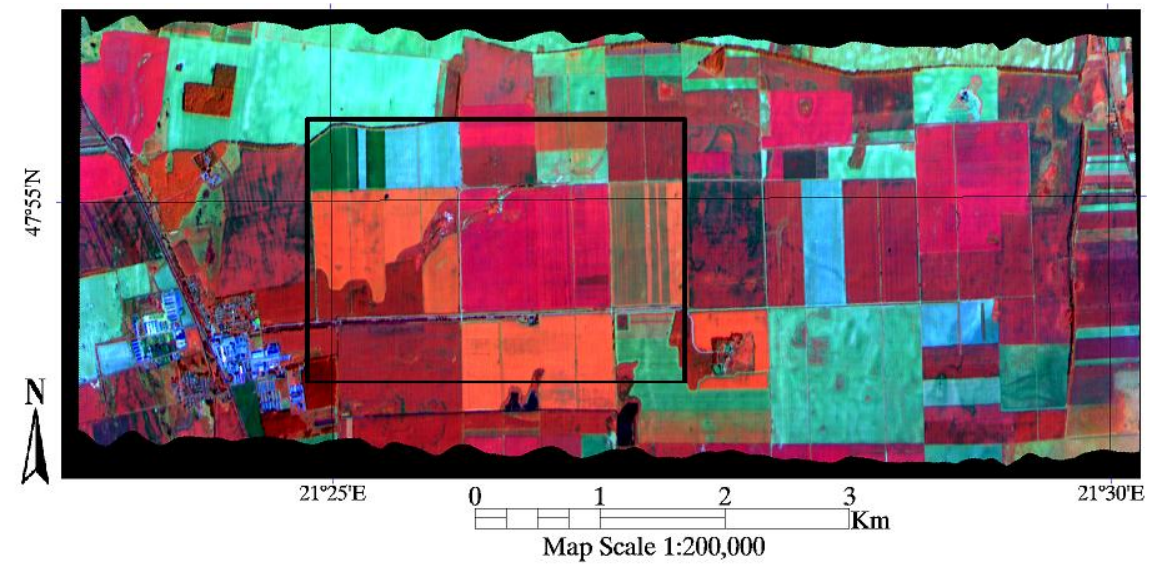

Fig. 2. Hyperspectral image of study area in Tedej, Hajdu-Bihar area, Hungary. Reflectance values for bands $29(0.988 \mu \mathrm{m}), 39(1.727 \mu \mathrm{m})$ and $1(0.496 \mu \mathrm{m})$. 
spatial resolution from 3-20 $\mathrm{m}$ depending on the carrier aircraft altitude [19]. The 79 channels consist of varying bandwidths which are useful for a large variety of applications.

Flights took place on the 17th and 18th August 2002. Absence of clouds and wind on the second day were appropriate and hence this image was used for the study. Atmospheric effects were minimized by applying an empirical line calibration method [24] to match light and dark measured field spectra targets. This was carried out by DLR German Aerospace Center and the processed data was made available. Therefore the details of atmospheric and image correction is not discussed in this paper. The study area is shown in Figure 1 and the hyperspectral image is shown in Figure 2 at $5 \mathrm{~m}$ nominal resolution on the ground.

Assuming that ground truth data are unavailable at the time of designing a sampling scheme, the bands selected were in close proximity to the wavelengths used in [30] to identify discriminating crops. The 18 bands selected were: $1(0.496 \mu \mathrm{m}), 4(0.551 \mu \mathrm{m}), 10(0.657 \mu \mathrm{m}), 11(0.675 \mu \mathrm{m}), 12(0.693 \mu \mathrm{m})$, $13(0.710 \mu \mathrm{m}), 14(0.727 \mu \mathrm{m}), 15(0.744 \mu \mathrm{m}), 23(0.886 \mu \mathrm{m}), 25(0.921 \mu \mathrm{m}), 29$ $(0.988 \mu \mathrm{m}), 32(1.035 \mu \mathrm{m}), 33(1.539 \mu \mathrm{m}), 37(1.668 \mu \mathrm{m}), 39(1.727 \mu \mathrm{m}), 49(2.084$ $\mu \mathrm{m}), 54(2.158 \mu \mathrm{m})$ and $59(2.232 \mu \mathrm{m})$. These include a series of visible, nearinfrared and short-wave-infrared bands. Bands 41-48 $(1.958-2.068 \mu \mathrm{m})$ and $62-$ $72(2.275-2.412 \mu \mathrm{m})$ were noisy and were not considered for selection since they could affect the results of the segmentation. Furthermore, the wavelengths of these bands are usually not considered as being very effective for discriminating between crops as opposed to other bands [30].

Even though we selected 18 bands for the image segmentation, the use of hyperspectral data has the advantage of producing better classification results over multispectral broadband data because of the narrow bands for the hyperspectral data $[29,3]$. Selection of bands becomes necessary when performing image segmentation on hyperspectral data because of the high correlations between the bands, thus often carrying redundant information [30].

Choice of spectral bands to use and restriction of categories to sample depends on the application and actual data. This is inevitable as relying solely on segmentation can result in sampling categories of no interest. Although bands selected in this study are not necessarily the optimal discriminating bands, they are commonly used for calculating the red-edge, vegetational indices or the amount of stress in vegetation. The segmented image thus inherit these qualities and resulted in an optimal prospective sampling scheme that best estimates the various vegetation indices.

The methodology is illustrated on a $600 \times 400$ pixel hyperspectral image, displayed as a black box in Figure 1, using the 18 spectral bands. The number of categories in the image is determined by executing an iterative procedure using the Bayesian Information Criteria (BIC) [13]. To avoid slow convergence and insufficient computer memory [13], a random sample of pixels was selected. The number of categories was determined repeatedly on subsets of the image for more stable results. The results indicate either seven or eight categories in the image. In this paper we used [2] iterated conditional modes algorithm (ICM) for image 
segmentation. Since ICM allows merging of categories by resulting in empty categories if they are similar, the algorithm was applied to eight categories. Other, more complex methods of determining the number of categories can be found in [28] and [12] using Pseudolikelihood Information Criteria (PLIC) instead of BIC. It was not intended here, however, to compare performance of these methods or the actual ICM algorithm.

\section{Methods}

\subsection{The ICM Algorithm}

Adequate image segmentation takes into account both spectral features and spatial information. Applications of the Markov Random Fields (MRF) have been useful in this respect [2]. The choice of ICM was rather subjective and various other segmentation methods are possible. A comparison of ICM with maximum likelihood classification and support vector machines can be found in [18], demonstrating comparable results between these three methods. Details concerning the ICM algorithm can be found in [2].

\subsection{Sampling Per Category}

The section describes the method for obtaining the optimal prospective sampling scheme within each category, after segmentation. Suppose that the segmented image at the $\alpha$ th iteration is denoted by $\mathbf{C}^{(\alpha)}=\bigcup_{k=1}^{K} \mathbf{C}_{k}^{(\alpha)}, \alpha=0,1,2, \ldots$, where $\mathbf{C}_{k}^{(\alpha)}$ denotes the set of pixels which belongs to the $k$ th category. Let $N_{k}^{(\alpha)}$ denote the number of elements in $\mathbf{C}_{k}^{(\alpha)}$, i.e. the number of pixels in the $k$ th category at the $\alpha$ th iteration. Also, let $f_{i j}$ denote the $m$-dimensional feature vector for pixel $(i, j)$, so $\mu_{k}^{(\alpha)}=\sum_{(i, j) \in \mathbf{C}_{k}^{(\alpha)}} f_{i j} / N_{k}^{(\alpha)}$ is the $m$-dimensional mean vector of the $k$ th category at the $\alpha$ th iteration.

Sample Size Per Category For a pre-specified number of $n$ samples, suppose a proportional allocation is assigned to each category $K$ [31]. An elementary adjustment to the formula presented in [31] was made to allow the minimum number of samples per category $n_{k}$ to be $n_{(0)}$ (see Equation 1 ) so that all categories are represented. The sample size per category is then obtained by distributing the remainder of the samples $\left(n-K \cdot n_{(0)}\right)$ proportionally according to the variability $\left(\nu_{k}^{(r)}\right)$ and size $\left(N_{k}^{(r)}\right)$ of the category at the $r$ th iteration. The sample size for category $k$ equals

$$
n_{k}=n_{(0)}+\left(n-K \cdot n_{(0)}\right) \cdot \frac{N_{k}^{(r)} \sqrt{\nu_{k}^{(r)}}}{\sum_{k=1}^{K} N_{k}^{(r)} \sqrt{\nu_{k}^{(r)}}},
$$


where $\nu_{k}^{(r)}=\frac{1}{N_{k}^{(r)}} \sum_{(i, j) \in \mathbf{C}_{k}^{(r)}}\left(f_{i j}-\mu_{k}^{(r)}\right)^{T}\left(f_{i j}-\mu_{k}^{(r)}\right)$.

Sampling is optimized over each category since segmentation is governed by categories, and sampling is carried out to characterize these categories. Choice of an appropriate optimization technique and a relevant criterion is of importance as the samples are to be spread over the category, consisting of several disjoint segments.

Simulated Annealing Per Category Simulated annealing is a general applicable optimization method to find the global optimum of an objective function in the presence of local optima. In simulated annealing, a fitness function $\phi(\mathbf{S})$, depending on the sampling configuration $\mathbf{S}$, has to be minimized. Details on simulated annealing as applied to sampling can be found in [32].

Fitness Function Per Category Various choices of fitness functions $\phi(\mathbf{S})$ can be made. For example, minimization of the Mean Shortest Distances (MMSD)criterion aims at even spreading of all sampling points over the sampling region by minimizing the expectation of the distance between an arbitrary chosen point and its nearest observation [32]. Each category is considered separately as a sampling region. The MMSD-criterion was chosen since it is able to spread the sampling points over each category, each category consisting of possibly several segments. In effect most segments in the image will also be sampled since the objective of this criterion will force sampling points to move to different segments, belonging to a common category, depending on the distance between nearby segments.

The initial sampling scheme for the $k$ th category $\mathbf{S}_{k}^{(0)}$ is a random selection of $n_{k}$ [see Equation 1] points from category $k$. For $\mathbf{S}_{k}$, the fitness function equals

$$
\phi_{\mathrm{MMSD}}\left(\mathbf{S}_{k}\right)=\frac{1}{N_{k}^{(r)}} \sum_{(i, j) \in \mathbf{C}_{k}^{(r)}}\left\|c_{k(i j)}-W_{\mathbf{S}_{k}}\left(c_{k(i j)}\right)\right\|,
$$

where $c_{k(i j)} \in \mathbf{C}_{k}^{(r)}$ is a location vector denoting the $(i, j)$ th pixel belonging to category $k$ and $W_{\mathbf{S}_{k}}\left(c_{k(i j)}\right)$ denotes the location vector of the nearest sampling point in $\mathbf{S}_{k}$.

Minimizing Equation 2 results in an even spreading of sampling points within category $k$, i.e. points will arrange at an equilateral triangular configuration. The final sampling scheme $\mathbf{S}$ consists of all sampling points in the $k$ categories, $\mathbf{S}=\bigcup_{k=1}^{K} \mathbf{S}_{k}$.

\section{Results}

ICM was applied to the subset hyperspectral data with the 18 selected bands described in section 2.2. The resulting segmented image with eight categories 


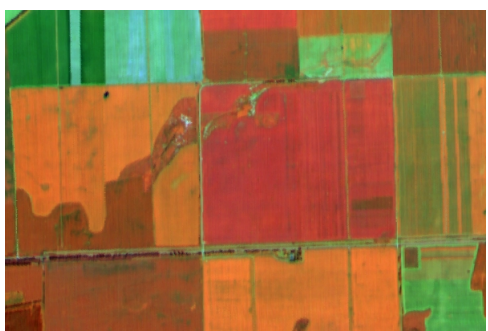

(a) Original hyperspectral image

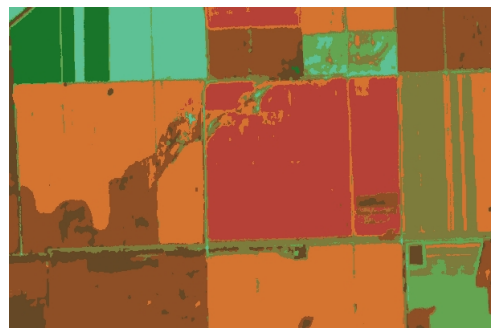

(b) ICM Segmented image with eight categories

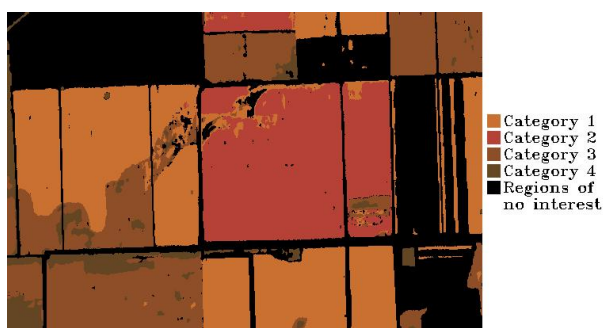

(c) Segmented image confining sampling regions to the four categories

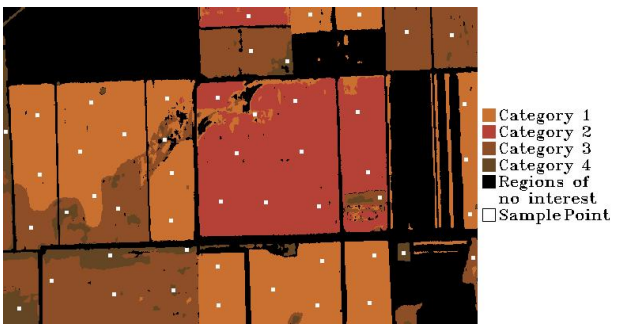

(d) Optimized sampling locations of 50 points distributed over 4 categories

Fig. 3. Methodology applied to DAIS-7915 hyperspectral image

is shown in Figure 3(b), which are the mean reflectance values for bands 29 $(0.988 \mu \mathrm{m}), 39(1.727 \mu \mathrm{m})$ and $1(0.496 \mu \mathrm{m})$. The sampling scheme was optimized by using the segmented image with eight categories. The image was first analyzed and adjustments were made to prevent sampling in areas of no interest. First, pixels belonging to pathways, between fields and non-vegetation categories (cultivated areas) were removed after segmentation but prior to sampling. Second, disjoint segments smaller than 10 pixels were removed from further analysis in the sampling stage. This decreased the chance of sampling in segments that were too heterogeneous or small in the image as it seemed impractical to sample in such areas. However, if these are meaningful segments, the above procedure can be performed without removal of these pixels. Four of the eight categories were cultivated crops and hence combined with pixels belonging to pathways between the fields to form a category that constrained sampling. The resulting image is shown in Figure 3(c) where sampling was optimized over categories 1-4.

As an illustration, a total sample size of 50 points was used. The number of samples for each category was then determined using Equation 1 by stipulating the minimum number of samples per category as 3. This resulted in 7, 11, 22 and 10 samples for each of the four categories. Samples for each category were then optimized by using simulated annealing and the MMSD-criterion as the fitness function. The distribution of the 50 sampling points is shown in Figure 3(d). In this image, the optimized prospective sample points tend to arrange in a trian- 
gular formation, while being governed by the actual complexity of the segments. Most samples are also arranged away from the borders of the segments.

\section{Validation}

This section demonstrates that the optimal prospective sampling scheme is suitable to estimate various vegetation parameters. It is known that the Leaf Area Index (LAI) is often used as a key variable in estimating foliage cover and forecasting vegetation growth and yield [16]. Several researchers have shown that there is a relationship between ground-measured LAI and vegetation indices [27, $1,7,8,11,4]$.

The reason for the varying vegetation indices is that different indices are used to capture different vegetation parameters of interest. Some indices are used to capture the photochemical processes associated with photosynthesis $[6,14,10,3$, $16]$, while others for retrieval of LAI estimates $[8,4,23]$, or to quantify vegetation fraction [15]. In all, vegetation indices are well correlated with various vegetation parameters such as LAI, biomass, chlorophyll concentration, and photosynthetic activity.

The Normalized Difference Vegetation Index (NDVI) developed by [26] is the most widely used vegetation index. In terms of the hyperspectral bands, NDVI is defined as

$$
\mathrm{NDVI}=\frac{R_{0.886}-R_{0.675}}{R_{0.886}+R_{0.675}}
$$

where $R_{x}$ is the reflectance of the wavelength at $x \mu \mathrm{m}$. The NDVI is based on the contrast between the maximum absorption in the red due to chlorophyll pigments to the maximum reflection in the infrared caused by leaf cellular structure. Figure 4(a) shows the NDVI image, where brighter pixels are indicative of healthy vegetation.

The Renormalized Difference Vegetation Index (RDVI) was developed by [25] to improve estimates of LAI. RDVI for the hyperspectral image is defined as

$$
\mathrm{RDVI}=\frac{R_{0.886}-R_{0.675}}{\sqrt{R_{0.886}+R_{0.675}}} .
$$

Figure 4(b) shows the RDVI image, where brighter pixels are indicative of healthy vegetation.

The Modified Simple Ratio (MSR) developed by [7] was also designed to improve estimates of LAI. MSR for the hyperspectral image is defined as

$$
\mathrm{MSR}=\left(\frac{R_{0.886}}{R_{0.675}}-1\right) / \sqrt{\frac{R_{0.886}}{R_{0.675}}+1} .
$$

Figure 4(c) shows the MSR image, where brighter pixels are indicative of healthy vegetation.

In attempting to improve the vegetation indices with regard to background soil, [22] modified the Soil-Adjusted Vegetation Index (MSAVI), which is defined 


$$
\mathrm{MSAVI}=\frac{1}{2}\left[\left(2 R_{0.886}+1\right)^{2}-8\left(R_{0.886}-R_{0.675}\right)\right] .
$$

Figure 4(d) shows the MSAVI image, where brighter pixels are indicative of healthy vegetation. Also noticeable is the region with high salinity, running diagonally across the center of the image, that has high values for MSAVI since the effect of the background soil is diminished.

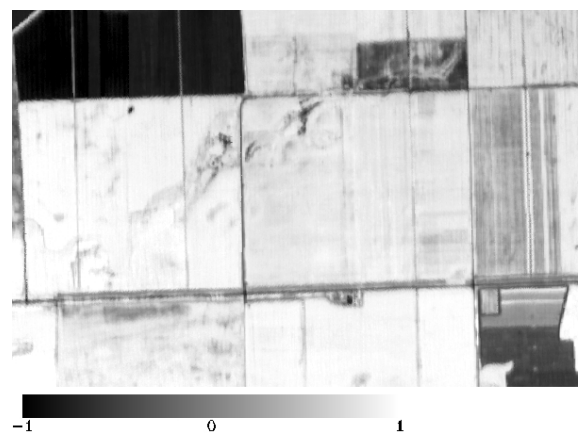

(a) NDVI

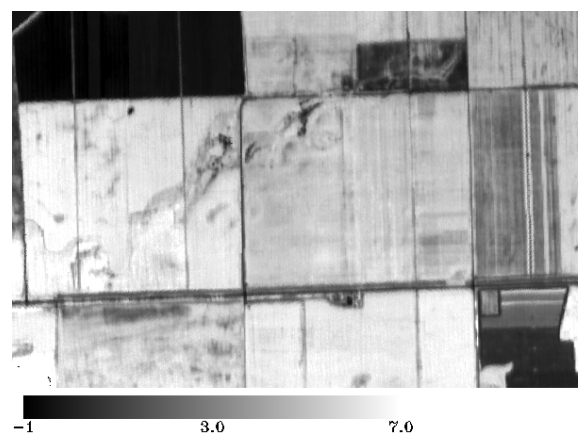

(c) MSR

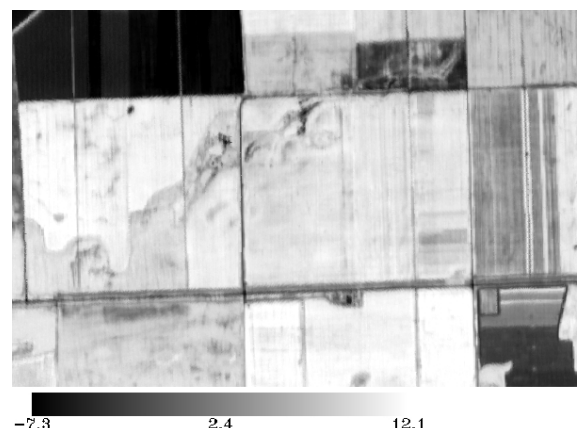

(b) RDVI

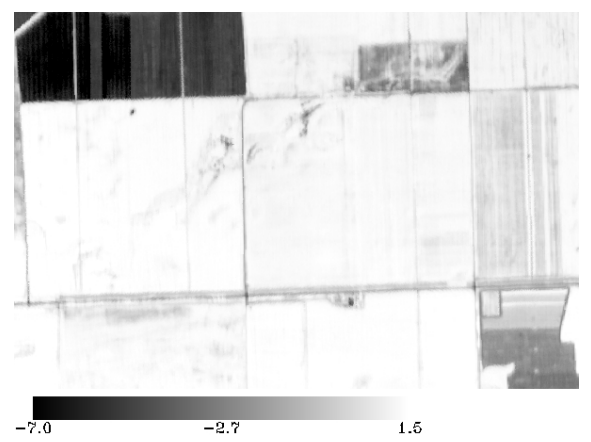

(d) MSAVI

Fig. 4. Vegetation indices maps.

The above four indices are used to show that the optimal prospective sampling scheme gives most precise estimates for each vegetation index compared to simple random sampling and grid sampling. For simple random sampling, three sampling schemes are designed, by randomly selecting 50 samples from the region of interest, to show inconsistency in the estimates. For the rectangular grid scheme, three sampling schemes were designed to again show inconsistency in the estimates. Initially, for each grid, 54 samples were selected, with a grid spacing of $300 \mathrm{~m}$. The first sample was randomly selected from a $300 \times 300 \mathrm{~m}$ grid. Nine samples were arranged in a row and six in a column, thus totalling to 
the 54 samples. For each grid sampling scheme, the samples falling in the noninterest area were removed. This resulted in either three or four samples removed from each grid sampling scheme. The average vegetation index for each sampling scheme is then calculated and compared to the respective average vegetation index for all pixels in the area of interest. The results are shown in Table 1. The optimized sampling scheme gives most accurate estimates for each vegetation index compared to the simple random and rectangular grid sampling schemes. Inconsistencies are apparent for each of the latter two sampling designs.

Table 1. Comparison of sampling schemes.

\begin{tabular}{lccccc}
\hline & \multicolumn{5}{c}{ Mean } \\
\cline { 2 - 6 } & & NDVI RDVI MSR MSAVI \\
\hline Image & 0.59 & 8.8 & 1.34 & 1.24 \\
Optimized sampling scheme & 0.58 & 8.6 & 1.32 & 1.22 \\
& & & & & \\
Random sampling scheme 1 & 0.49 & 7.9 & 1.18 & 1.09 \\
& 2 & 0.38 & 6.1 & 0.94 & 0.89 \\
& 3 & 0.45 & 7.0 & 1.11 & 1.06 \\
Grid sampling scheme & 1 & 0.49 & 7.8 & 1.14 & 1.13 \\
& 2 & 0.53 & 8.2 & 1.25 & 1.13 \\
& 3 & 0.53 & 8.3 & 1.26 & 1.15 \\
\hline
\end{tabular}

Values in the table represent the average vegetation indices for each sampling scheme.

\section{Discussion}

The sampling methodology presented in this paper was tested on a subset of a DAIS-7915 hyperspectral image by initially applying the ICM algorithm. The samples per category were obtained in proportion to the size and variability of the category as fewer samples are required to estimate smaller, more homogenous categories. The assumption that categories, comprising of possibly several segments, are homogeneous, logically makes it possible to spread sampling points over each category by using the MMSD-criterion as the fitness function in simulated annealing. Although simulated annealing is a slow process, it arrives at the global optimum. Disjoint segments for each category results in many local minima for the fitness function and hence necessitates such an algorithm. Most of the samples are away from the boundaries of these categories where uncertainty is high. This highlights the appropriateness of the implemented fitness function. This is further supported by samples spread over the categories, forming an equilateral triangular structure. It depended though on the spatial complexity 
of the category. Since these sampling points are geo-referenced, the location can be determined for field visits at these sampling points on the ground.

Field spectral measurements of agricultural crops could be used, for example, to study health of crops and thus are important for estimating foliage cover and forecasting vegetation growth and yield. This necessitates regular field visits. Because fields of agricultural crops may be homogeneous (planted with only one particular type of crop) or heterogeneous (planted with a group of different types of crops), the lack of this prior information can make field sampling non-optimal. Classification of agricultural fields is therefore useful in the optimization of prospective sampling schemes to support provisions that will sustain economic crop productivity. This hypothesis converges with [31] suggestion that estimation of the population mean may benefit from partitioning a study area into homogeneous strata.

Using appropriate band ratios, commonly used vegetation index maps were used to compare the optimal prospective sampling scheme to simple random sampling and rectangular grid sampling schemes. The optimal prospective sampling scheme results in estimates for each of the four vegetation indices considered that are closest to the actual averages for all pixels in the image. The rectangular grid sampling schemes also have reasonably accurate estimates but depends highly on the initial random sample. Inconsistencies between each rectangular grid sampling scheme can be observed. The estimates are poor using simple random sampling and inconsistencies for these sampling schemes are high. The optimized sample points therefore represent the whole study area more accurately than either of the other two sampling schemes for each of the different parameters of interest.

The ICM algorithm has regularly been applied for various purposes as it is quick and produces reasonably accurate categories. The drawback is that it may arrive at a local optimum, hence emphasizing the choice of initial means. For this reason, the $K$-means multivariate clustering algorithm was used as opposed to randomly selecting points in the image to represent the means for each category.

Sampling of categories is not restrictive to the use of the ICM algorithm and in this sense any other segmentation method may be used. This methodology uses remote sensing in designing optimal prospective sampling schemes on the ground for field visits as opposed to the traditional way of selecting samples randomly or on the basis of disciplinary judgement. The selected samples will in effect have image characteristics, such as, gray tone, texture or pattern, depending on the type of segmentation performed.

\section{Conclusions}

This study resulted into two main conclusions.

- The combination of the ICM algorithm for image segmentation and simulated annealing for optimized sampling provides an elegant and powerful tool in designing prospective sampling schemes using hyperspectral data. 
- The optimized prospective sampling scheme shows superiority, in this case study, to simple random sampling and rectangular grid sampling in estimating common vegetation indices and is thus more representative of the whole study area.

Acknowledgment The authors would like to acknowledge thanks to the sponsors of this work, namely, ITC International Institute for Geo-Information Science and Earth Observation, project number 3083022 and NRF National Research Foundation SA, project number 10317, gun 2053944.

\section{References}

[1] Baret, F., Guyot, G.: Potentials and limits of vegetation indices for LAI and APAR assessment. Remote Sensing of Environment, 35, 161-173 (1991).

[2] Besag, J.: On the statistical analysis of dirty pictures. Royal Statistical Society, B-48(3), 259-302 (1986).

[3] Broge, N.H., Leblanc, E.: Comparing prediction power and stability of broadband and hyperspectral vegetation indices for estimation of green leaf area index and canopy chlorophyll density. Remote Sensing of Environment, 76, 156-172 (2000).

[4] Brown, L., Jin, M.C., Lablanc, S.G., Cihlar, J.: A shortwave infrared modification to the simple ratio for LAI retrieval in boreal forests: An image and model analysis. Remote Sensing Environment, 71, 16-25 (2000).

[5] Brus, D.J., Spatjens, L.E.E.M., de Gruitjer, J.J.: A sampling scheme for estimating the mean extractable phosphorus concentration of fields for environmental regulation. Geoderma, 89, 129-148 (1999).

[6] Chappelle, E.W., Kim, M.S., McMurtrey III, J. E.: Ratio analysis of reflectance spectra (RARS): An algorithm for the remote estimation of the concentrations of chlorophyll a, chlorophyll b, and the carotenoids in soybean leaves. Remote Sensing of Environment, 39, 239-247 (1992).

[7] Chen, J.: Evaluation of vegetation indices and modified simple ratio for boreal applications. Canadian Journal of Remote Sensing, 22, 229-242 (1996).

[8] Chen, J., Cihlar, J.: Retrieving leaf area index of boreal conifer forests using Landsat Thematic Mapper. Remote Sensing of Environment, 55, 153-162 (1996).

[9] Curran, P.J., Williamson, H.D.: The accuracy of ground data used in remote-sensing investigations. International Journal of Remote Sensing, 6(10), 1637-1651 (1985).

[10] Daughtry, C.S.T., Walthall, C.L., Kim, M.S., Brown de Colstoun, E., McMurtrey III, J.E.: Estimating corn leaf chlorophyll concentration from leaf and canopy reflectance. Remote Sensing of Environment, 74, 229-239 (2000).

[11] Fassnacht, K.S., Gower, S.T., MacKenzie, M.D., Nordheim, E.V., Lillesand, T.M.: Estimating the leaf area index of north central Wisconsin forest using Landsat Thematic Mapper. Remote Sensing Environment, 61, 229-245 (1997).

[12] Forbes, F., Peyrard, N.: Hidden Markov Random Field Model selection criteria based on mean field-like approximations. IEEE Transactions on Pattern Analysis and Machine Intelligence, 25(9), 1089-1101 (2003).

[13] Fraley, C., Raftery, A.E. Model-Based Clustering for Image Segmentation and Large Datasets Via Sampling. Technical Report 424, University of Washington, Department of Statistics (2003). 
[14] Gamon, J.A., Penuelas, J., Field, C.B.: A narrow-waveband spectral index that tracks diurnal changes in photosynthetic efficiency. Remote Sensing of Environment, 41, 35-44 (1992).

[15] Gitelson, A., Rundquist, D., Derry, D., Ramirez, J., Keydan, G., Stark, R., Perk, R.: Using remote sensing to quantify vegetation fraction in corn canopies. In Proceedings of Third Conference on Geospatial Information in Agriculture and Forestry, pp. 3-7, Denver, Colorado, November 2001 (2001).

[16] Haboudanea, D., Millera, J.R., Patteyc, E., Zarco-Tejadad, P.J., Strachane, I.B.: Hyperspectral vegetation indices and novel algorithms for predicting green LAI of crop canopies: Modeling and validation in the context of precision agriculture. Remote Sensing of Environment, 90, 337-352 (2004).

[17] Kardeván, P., Vekerdy, Z., Róth, L., Sommer, S., Kemper, T., Jordan, G., Tamás, J., Pechmann, I., Kovács, E., Hargitai, H., László, F.: Outline of scientific aims and data processing status of the first Hungarian hyperspectral data acquisition flight campaign, HYSENS 2002 Hungary. In Habermeyer, M., Mülle, A., Holzwarth, S. (eds.), Proceedings of the 3rd EARSeL workshop on imaging spectroscopy, pp. 324332, Herrsching, Germany: EARSeL (2003).

[18] Keuchel, J., Naumann, S., Heiler, M., Siegmund, A.: Automatic land cover analysis for Tenerife by supervised classification using remotely sensed data. Remote Sensing of Environment, 86, 530-541 (2003).

[19] Lehmann, F., Oertel, D., Richter, R., Rothfuss, H., Strobl, P., Muller, A., Tischler, S., Mueller, R., Beran, D., Fries, J., Boehl, R., Obermeier, P.: Hyperspectral applications with a new sensor. Presentation at the ISSSR (International Symposium on Spectral Sensing Research) in Melbourne: DAIS-7915, The Digital Airborne Imaging Spectrometer DAIS-7915 (1995).

[20] Lu, D., Mausel, P., Brondízio, E., Moran, E.: Classification of successional forest stages in the Brazilian Amazon basin. Forest Ecology and Management, 181(3), 301-312 (2003).

[21] Moreau, M., Laffly, D., Joly, D., Brossard, T.: Analysis of plant colonization on an arctic moraine since the end of the Little Ice Age using remotely sensed data and a Bayesian approach. Remote Sensing of Environment, 99, 244-253 (2005).

[22] Qi, J., Chehbouni, A., Huete, A.R., Keer, Y.H., Sorooshian, S.: A modified soil vegetation adjusted index. Remote Sensing of Environment, 48, 119-126 (1994).

[23] Qi, J., Kerr, Y.H., Moran, M.S., Weltz, M., Huete, A.R., Sorooshian, S., Bryant, R.: Leaf area index estimates using remotely sensed data and BRDF models in a semiarid region. Remote Sensing of Environment, 73, 18-30 (2000).

[24] Roberts, D.A., Yamaguchi, Y., Lyon, R.J.P.: Calibration of airborne imaging spectrometer data to percentage reflectance using field spectral measurements. In Proceedings of the Nineteenth International Symposium on Remote Sensing of the Environment, pp. 21-25: Ann Arbor, Michigan (1985).

[25] Rougean, J.L., Breon, F.M.: Estimating PAR absorbed by vegetation from bidirectional reflectance measurements. Remote Sensing of Environment, 51, 375-384 (1995).

[26] Rouse, J.W., Haas, R.H., Schell, J.A., Deering, D.W., Harlan, J.C.: Monitoring the vernal advancements and retrogradation of natural vegetation. In NASA/GSFC, Final Report, Greenbelt, MD, USA, pp. 1-137 (1974).

[27] Spanner, M.A., Pierce, L.L., Peterson, D.L., Running, S.W.: Remote sensing of temperate coniferous forest leaf area index: The influence of canopy closure, understory vegetation and background reflectance. Internation Journal of Remote Sensing, 11, 95-111 (1990). 
[28] Stanford, D.C., Raftery, A.E.: Approximate bayes factors for image segmentation: The pseudolikelihood information criterion (PLIC). IEEE Transactions on Pattern Analysis and Machine Intelligence, 24(11), 1517-1520 (2002).

[29] Thenkabail, P. S., Enclonab, E.A., Ashton, M.S., Legg, C., De Dieu, M.J.: Hyperion, IKONOS, ALI, and ETM+ sensors in the study of African rainforests. Remote Sensing of Environment, 90, 23-43 (2004).

[30] Thenkabail, P.S.: Optimal hyperspectral narrowbands for discriminating agricultural crops. Remote Sensing Reviews, 20(4), 257-291 (2002).

[31] Thompson, S.K.: Sampling. John Wiley and Sons, Inc., NY (1992).

[32] Van Groenigen, J.W., Stein, A.: Constrained optimization of spatial sampling using continuous simulated annealing. Journal Environmental Quality, 27, 1078-1086 (1998).

[33] Watson, F.G.R., Anderson, T.N., Newman, W.B., Alexander, S.E., Garrott, R.A.: Optimal sampling schemes for estimating mean snow water equivalents in stratified heterogeneous landscapes. Journal of Hydrology, 328, 432-452 (2006). 